\title{
Desempenho do Irrigâmetro na estimativa da evapotranspiração de referência
}

\author{
Rubens A. Oliveira ${ }^{1}$, Cristiano Tagliaferre ${ }^{2}$, Gilberto C. Sediyama ${ }^{1}$, Franklin J. V. Materam ${ }^{3}$ \& Paulo R. Cecon ${ }^{4}$
}

\begin{abstract}
RESUMO
Neste trabalho objetivou testar o Irrigâmetro para estimar a evapotranspiração de referência comparativamente com o método de Penman-Monteith - FAO 56, e confrontar o seu desempenho com os métodos Penman modificado - FAO 24, Radiação - FAO 24, tanque Classe A e Hargreaves-Samani (1985). O Irrigâmetro é um aparelho evapo-pluviométrico desenvolvido recentemente na Universidade Federal de Viçosa para ser utilizado no manejo da irrigação. Os dados de evapotranspiração de referência (ETo) obtidos no aparelho foram confrontados com os dados obtidos pelo método de Penman-Monteith, em intervalos de um, três, cinco e sete dias. Conforme os resultados, o Irrigâmetro apresentou desempenho satisfatório para estimar a ETo em todos os intervalos de tempo estudados. Este desempenho, associado ao baixo custo, à alta praticidade e à inexistência de cálculos, torna o aparelho indicado para o manejo da água na agricultura irrigada. Os métodos de Penman modificado, Radiação e Hargreaves-Samani, superestimaram os valores da ETo obtidos pelo método de Penman-Monteith, tendo o tanque Classe A apresentado comportamento oposto.
\end{abstract}

Palavras-chave: evaporação, evaporímetro, manejo da água de irrigação

\section{Performance of the "Irrigâmetro" in the estimation of reference evapotranspiration}

\begin{abstract}
The objective of this work was to test the "Irrigâmetro" to estimate the reference evapotranspiration in comparison to the Penman-Monteith method - FAO 56, and to compare its potential with the modified methods of Penman - FAO 24, Radiation - FAO 24, Class A Pan and Hargreaves-Samani (1985). The Irrigameter is an evapo-pluviometric apparatus recently developed at the Federal University of Viçosa to be used in irrigation water management. The reference evapotranspiration data obtained from the apparatus are compared with the data obtained from the method of PenmanMonteith, in intervals of one, three, five and seven days. According to the results, the Irrigameter presented satisfactory data to estimate ETo in all time intervals studied. Its low cost, high practicairty and inexistence of any additional calculations make it very suitable for irrigation water management in agriculture. The methods of modified Penman, Radiation and Hagreaves-Samani overestimated the ETo values obtained by the method of Penman-Monteith, while the Class A Pan presented the opposite behavior.
\end{abstract}

Key words: evaporation, evaporimeter, irrigation water management

1 DEA/UFV, CEP 36570-000, Viçosa, MG. Fone: (31) 3899-1909; (31) 3899-2729. E-mail: Rubens@ufv.br; g.sediyama@ufv.br

${ }^{2}$ DEAS/UESB. Fone: (77) 8815-8579. E-mail: tagliaferre@yahoo.com.br

3 Universidad Del Zúlia/Venezuela. E-mail: franklinvalbuena@yahoo.com.br

${ }^{4}$ DPI/UFV. Tel. (31) 3899-1781. E-mail: cecon@dpi.ufv.br 


\section{INTRODUÇÃO}

A água é indispensável à vida, sendo um recurso natural limitado e dotado de valor econômico, sua escassez, tanto em nível nacional quanto mundial, está aumentando a competição entre os diferentes usuários, como indústria, abastecimento humano e agricultura.

No Brasil, a agricultura irrigada consome uma grande parcela da água usada nas diversas atividades humanas, razão por que se ressalta que, na maioria das áreas irrigadas, é comum observar ausência de manejo racional da água resultando, geralmente, em aplicação excessiva, com desperdício de água e energia, além da ocorrência de problemas ambientais, ou em deficiência hídrica para as plantas, com baixa produtividade e prejuízos econômicos ao produtor. Práticas adequadas de manejo da irrigação contribuem para aumentar a produtividade e melhorar a qualidade dos produtos agrícolas, para minimizar o uso de água e preservar os recursos hídricos.

O manejo da água de irrigação é, em geral, conduzido através da avaliação da umidade do solo ou por meio de estimativa da evapotranspiração da cultura, sendo este método mais usado em virtude da sua maior praticidade e da menor exigência de mão-de-obra.

No manejo da irrigação, conduzido através de estimativa de evapotranspiração, os equipamentos mais usados são o tanque Classe A e a estação meteorológica automática, em que o tanque Classe A é um dos métodos mais utilizados para estimativa da ETo no manejo da irrigação e se baseia na medição da evaporação da água em um tanque padronizado, cujo valor é convertido em evapotranspiração de referência por meio de coeficientes específicos dependentes do clima, do tipo de tanque e da bordadura circundante (Doorenbos \& Pruitt, 1977; Allen et al., 1998). Segundo Sediyama (1996), o maior uso desse equipamento se deve à sua praticidade e aos baixos custos de instalação e manutenção. De acordo com Smith (1991), quando bem conduzido, este método oferece resultados confiáveis na determinação da evapotranspiração de referência.

No caso de uso do tanque Classe A, a medição da lâmina de água evaporada e sua conversão em evapotranspiração exigem que o irrigante faça cálculos muito simples e tenha certo conhecimento técnico sobre irrigação, o que tem dificultado seu uso generalizado no manejo da água na agricultura irrigada. Este aspecto relativo ao uso do tanque Classe A constitui uma desvantagem haja vista que dificulta a tomada de decisão do irrigante quanto ao momento de efetuar a próxima irrigação e em relação à quantidade de água a ser aplicada, o que interfere na definição do tempo de funcionamento do equipamento de irrigação.

No caso de uso de estação meteorológica automática, estão associados elevados custos para o produtor envolvendo a aquisição da estação, de computador e de programa computacional, manutenção periódica, assistência técnica especializada, além de certo conhecimento técnico do irrigante a respeito de irrigação. Esses custos associados inviabilizam o uso de estação meteorológica automática para a grande maioria dos produtores que trabalham no âmbito da agricultura irrigada no Brasil.
Ley et al. (1994a e 1994b) citam que, quando a medição dos elementos meteorológicos é feita por estações meteorológicas automáticas, é comum a ocorrência de erros decorrentes da calibração dos sensores dos equipamentos utilizados para essas medições comprometendo, desta forma, a determinação dos componentes necessários à estimativa da evapotranspiração. Segundo os autores, quando as medições advêm de estações meteorológicas convencionais, os erros são ainda maiores pois se acrescentam, aos erros instrumentais, os erros devidos ao observador.

O Irrigâmetro é um aparelho inventado recentemente na Universidade Federal de Viçosa, detentora de sua patente, trata-se de um aparelho evapo-pluviométrico a ser utilizado no manejo da irrigação visando otimizar o uso da água na agricultura irrigada. Esta otimização é importante para a sociedade como um todo, tanto do ponto de vista ambiental, economizando água e energia e evitando degradação do meio ambiente, quanto do ponto de vista de geração de renda para o produtor rural, reduzindo custos e aumentando a produtividade das culturas e a disponibilidade de alimentos de melhor qualidade.

O Irrigâmetro, ao introduzir grande simplicidade no manejo da água em áreas irrigadas, constitui-se em um aparelho de grande potencial de uso na agricultura irrigada uma vez que, além de diversas vantagens, ele fornece resposta prática às duas perguntas básicas do manejo de irrigação: quando e quanto irrigar.

Em condições normais de manejo da irrigação com o tanque Classe A, o operador mede a lâmina de água evaporada e a lâmina precipitada, com uso de pluviômetro e, posteriormente, efetua os cálculos necessários para determinar quando e quanto irrigar, por outro lado, em condições de manejo da irrigação com uso do Irrigâmetro, a informação relativa ao momento e ao tempo de funcionamento do equipamento de irrigação, ou a sua velocidade de deslocamento, fica prontamente disponível ao irrigante, que não precisa ter conhecimento técnico avançado em irrigação nem efetuar cálculos. No caso de ocorrência de chuva, a lâmina precipitada é medida e computada facilmente pelo operador do Irrigâmetro sabendo-se, em seguida, se ela foi suficiente ou não para suprir o déficit hídrico até então existente no solo, também sem a necessidade de fazer cálculos, os quais, muitas vezes, são desconhecidos por alguns irrigantes brasileiros.

Dependendo das dimensões de componentes do Irrigâmetro, tais como a área do evaporatório e a do tubo de alimentação, a medição da lâmina evaporada ou evapotranspirada pode ser feita com alta precisão. A alta precisão na leitura da lâmina é particularmente importante na condução de pesquisas científicas aplicadas na área de Agrometeorologia e de Ciência da Irrigação, relacionadas à determinação de demanda hídrica das culturas. Para fins práticos de manejo da irrigação, a medição da lâmina com precisão de um décimo de milímetro é plenamente satisfatória.

O Irrigâmetro é de custo relativamente baixo, de aproximadamente $\mathrm{R} \$ 1.590,00$ e de fácil operação a qual consiste simplesmente em abertura e fechamento de válvulas nele existentes, obedecendo a uma seqüência previamente definida. 
Neste trabalho o objetivo principal foi testar o Irrigâmetro com o método de Penman-Monteith - FAO 56, para estimativa da evapotranspiração de referência (ETo) e confrontar seu desempenho com os métodos Penman modificado FAO 24, Radiação - FAO 24, tanque Classe A e Hargreaves \& Samani (1985).

\section{MATERIAL E MÉTODOS}

O estudo foi conduzido na Unidade de Pesquisa e Desenvolvimento do Irrigâmetro, pertencente ao Departamento de Engenharia Agrícola da Universidade Federal de Viçosa, em Viçosa, MG, situada a $20^{\circ} 45^{\prime}$ de latitude Sul e $42^{\circ} 51^{\prime}$ de longitude Oeste, numa altitude média de $651 \mathrm{~m}$. Os elementos climáticos diários foram obtidos durante 42 dias, na estação climatológica do INMET (Instituto Nacional de Meteorologia) localizada no Campus da UFV, próximo à área experimental.

O Irrigâmetro é um aparelho que pode ser usado para estimar a evaporação, a evapotranspiração de referência (no caso deste estudo) e a evapotranspiração da cultura nas suas diversas fases de desenvolvimento, possui um evaporatório, no qual o nível da água é mantido constante através de um sistema semelhante ao usado no frasco de Mariotte; quatro válvulas que permitem efetuar o abastecimento de água, o escapamento de ar, a drenagem e a interconexão do tubo de alimentação com o evaporatório, além de três escalas indicadoras da leitura da lâmina evaporada ou evapotranspirada, do tempo de irrigação ou da velocidade de rotação do sistema de irrigação, e do momento de irrigar. O Irrigâmetro possibilita que se compute a efetividade da chuva sem necessidade de cálculos. A metodologia de funcionamento e suas partes constituintes podem ser encontrada em Oliveira \& Tagliaferre (2006).

A evapotranspiração de referência foi medida no Irrigâmetro com escala de leitura ampliada em 15 vezes e com resolução igual a 0,1 mm. A Figura 1 mostra o Irrigâmetro instalado na área experimental com a escala da lâmina evapotranspirada e as réguas que fornecem diretamente o tempo de irrigação, para

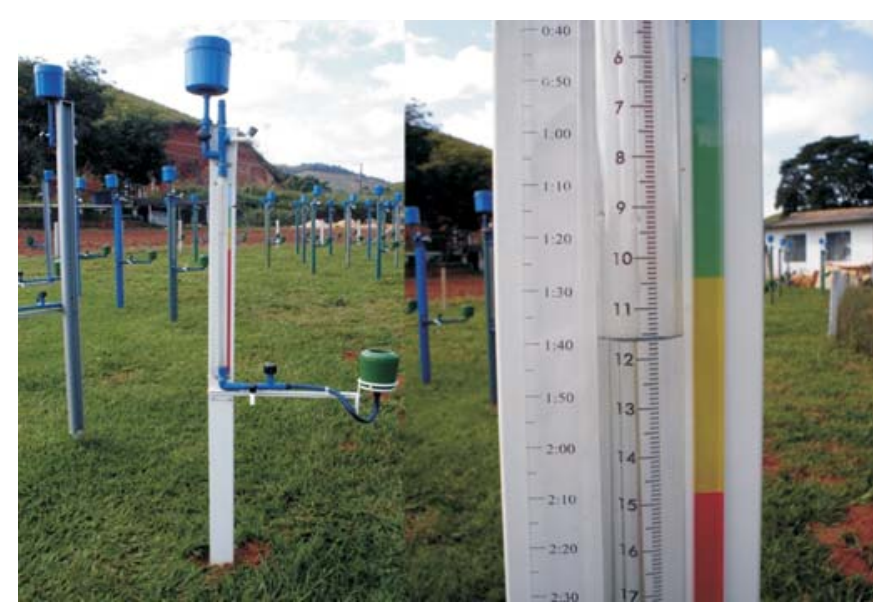

Figura 1. Equipamento instalado na Unidade de Pesquisa e Desenvolvimento do Irrigâmetro, pertencente ao Departamento de Engenharia Agrícola da Universidade Federal de Viçosa. No detalhe se observam a indicação da lâmina evapotranspirada $(11,6$ mm), o tempo de irrigação $(1: 40 \mathrm{~h})$ e o momento de irrigar (cor amarela) certo sistema de aspersão convencional, e o momento de irrigar, para determinado tipo de cultura e de solo.

A estimativa da ETo foi realizada através do Irrigâmetro, dos métodos propostos pela FAO (Penman-Monteith - Padrão FAO 56, Penman modificado - FAO 24, Radiação FAO 24, do tanque Classe A) e do método de Hargreaves \& Samani (1985). O método de Penman-Monteith foi adotado como padrão para testar a ETo com os demais métodos, conforme proposto por Smith (1991) e Allen et al. (1998).

Obtiveram-se os valores de ETo estimados pelos métodos de Penman-Monteith - FAO 56, Penman modificado, Radiação e Hargreaves-Samani com uso do aplicativo computacional REF-ET (Allen, 2000).

Fez-se a análise do desempenho do Irrigâmetro comparando-se os valores de evapotranspiração de referência, obtidos no aparelho, com os estimados pelo método de PenmanMonteith - FAO 56. A metodologia adotada para comparação dos resultados foi proposta por Allen (1986), e se fundamenta na estimativa do erro-padrão (EEP), calculada pela equação:

$$
\operatorname{EEP}=\left(\frac{\sum(\mathrm{y}-\hat{\mathrm{y}})^{2}}{\mathrm{n}-1}\right)^{1 / 2}
$$

em que:

$$
\begin{aligned}
\text { EEP - } & \text { estimativa do erro padrão, } \mathrm{mm} \mathrm{d}^{-1} \\
\mathrm{y}- & \text { evapotranspiração de referência estimada pelo } \\
& \text { método-padrão, } \mathrm{mm} \mathrm{d}^{-1} \\
\hat{\mathrm{y}}- & \text { evapotranspiração de referência obtida no Irri- } \\
& \text { gâmetro, mm d } \mathrm{d}^{-1} \\
\mathrm{n} \text { - } & \text { número de observações }
\end{aligned}
$$

A hierarquização das estimativas da evapotranspiração foi feita com base nos valores de estimativa do erro padrão, do coeficiente de determinação $\left(\mathrm{r}^{2}\right)$ e do coeficiente angular (b) das respectivas regressões lineares simples. A melhor alternativa foi aquela que apresentou maior $\mathrm{r}^{2}$, menor EEP e b próximo da unidade.

A precisão foi dada pelo coeficiente de determinação, a qual indica o grau em que a regressão explica a soma do quadrado total. A aproximação dos valores de ETo estimados por determinado método estudado, em relação aos valores obtidos com uso do método padrão, foi obtida por um índice designado concordância ou ajuste, representado pela letra "d" (Willmott et al., 1985), seus valores variam desde zero, onde não existe concordância, a 1, para a concordância perfeita.

O índice de aproximação é calculado aplicando-se a seguinte expressão:

$$
\mathrm{d}=1-\frac{\sum_{\mathrm{i}=1}^{\mathrm{n}}(\mathrm{Pi}-\mathrm{Oi})^{2}}{\sum_{\mathrm{i}=1}^{\mathrm{n}}[(|\mathrm{Pi}-\overline{\mathrm{O}}|)+(|\mathrm{Oi}-\overline{\mathrm{O}}|)]^{2}}
$$

em que:

d - índice de concordância ou ajuste

Pi - evapotranspiração de referência obtida pelo método considerado, $\mathrm{mm} \mathrm{d}^{-1}$

Oi - evapotranspiração de referência obtida pelo método-padrão, $\mathrm{mm} \mathrm{d}^{-1}$

$\overline{\mathrm{O}}$ - média dos valores de ETo obtida pelo métodopadrão, mm d $\mathrm{m}^{-1}$

$\mathrm{n}$ - número de observações 


\section{RESULTADOS E DISCUSSÃO}

Na Figura 2 se encontram as variações médias pentadiais dos valores dos elementos meteorológicos usados na estimativa da evapotranspiração de referência, durante o período estudado.

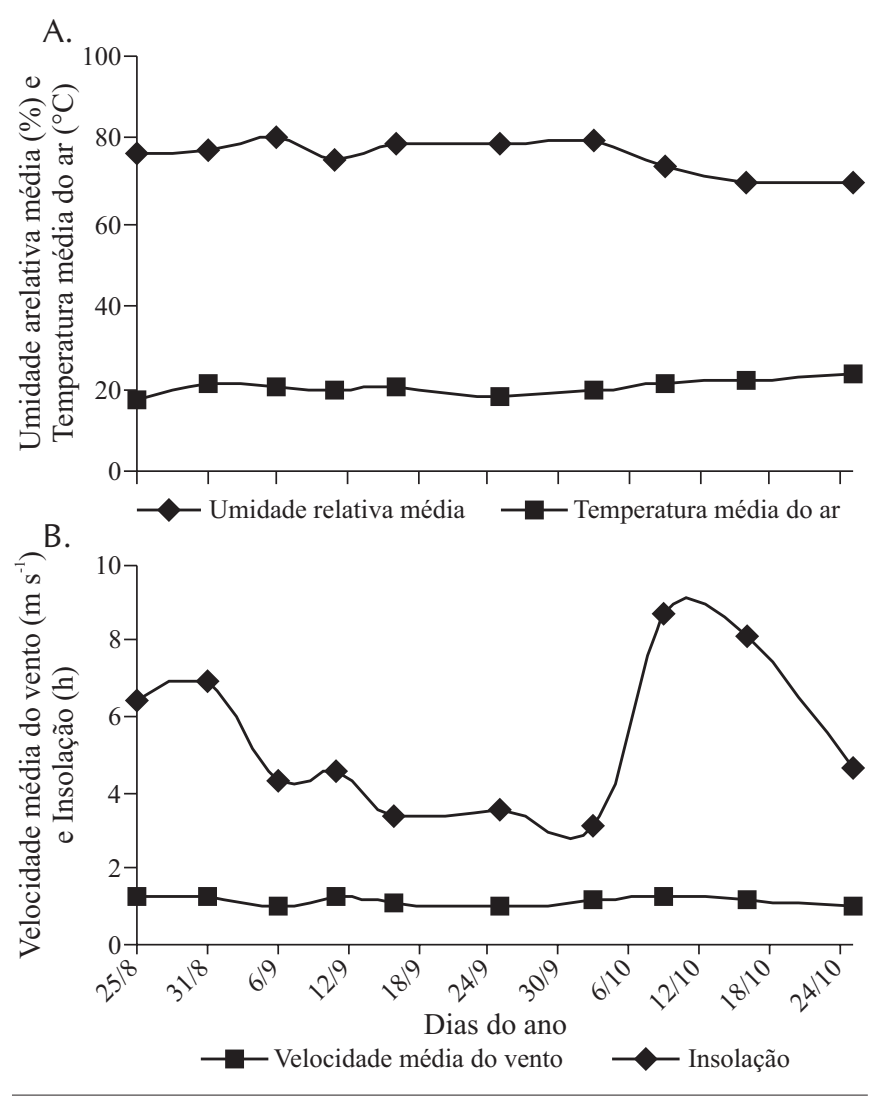

Figura 2. Variação pentadial da umidade relativa do ar e da temperatura do ar (Figura A) e da velocidade média do vento e da insolação (Figura B)

Na Figura 2 se encontram os elementos climáticos que influenciam diretamente a evapotranspiração, essenciais para o uso do método de Penman-Monteith - FAO 56, considerado padrão. O Irrigâmetro é um aparelho que possibilita diretamente a obtenção da evapotranspiração de referência não se necessitando, portanto, de medição de elementos climáticos nem de cálculos ou uso de programas computacionais.

Notou-se, durante a condução do experimento, pequena variação nos valores dos elementos climáticos, exceto a insolação. Na Figura 2A, a umidade relativa média diária foi superior a 70\%, classificada alta, e a temperatura média diária foi $20,5^{\circ} \mathrm{C}$, enquanto na Figura $3 \mathrm{~B}$ a velocidade média do vento foi inferior a $2 \mathrm{~m} \mathrm{~s}^{-1}$, considerada leve, de acordo com Doorenbos \& Pruitt (1977).

Na Tabela 1 se encontram os parâmetros da regressão, o coeficiente de determinação $\left(\mathrm{r}^{2}\right)$, a estimativa do erro padrão (EEP) e o coeficiente de ajustamento (d), obtidos das correlações entre os valores de $\mathrm{ET}_{0}$ estimada no Irrigâmetro e pelos demais métodos estudados, com valores estimados pelo método de Penman-Monteith - FAO 56, para períodos diários.

Conforme a Tabela 1 o método de Penman modificado, que leva em consideração os aspectos aerodinâmicos e de radia-
Tabela 1. Parâmetros da regressão, coeficiente de determinação $\left(\mathrm{r}^{2}\right)$, estimativa do erro padrão (EEP) e o coeficiente de ajustamento (d), obtidos das correlações entre os valores de ETo $\left(\mathrm{mm} \mathrm{d}^{-1}\right)$ estimada no Irrigâmetro e pelos demais métodos estudados, com valores estimados pelo método de Penman-Monteith - FAO 56, para períodos diários

\begin{tabular}{lcccccc}
\hline Método & $\mathbf{b}_{\mathbf{0}}=\mathbf{0}$ & $\mathbf{b}_{\mathbf{1}}=1$ & $\mathbf{r}^{2}$ & EEP & $\mathbf{d}$ & ETo \\
P. -Monteith - FA0 56 & & & & & & 3,24 \\
Penman modificado & $-0,917^{*}$ & $1,348^{*}$ & 0,98 & 0,44 & 0,96 & 3,45 \\
Hargreaves-Samani & $1,574^{*}$ & $0,839^{*}$ & 0,76 & 1,17 & 0,75 & 4,29 \\
Radiação & $-1,033^{*}$ & $-1,429^{*}$ & 0,97 & 0,60 & 0,94 & 3,60 \\
Tanque Classe A & - & $0,876^{*}$ & 0,98 & 0,62 & 0,89 & 2,86 \\
Irrigâmetro & - & $1,056^{*}$ & 0,98 & 0,53 & 0,93 & 3,44 \\
\hline * Significativo a 5\% de probabilidade pelo teste t & & & &
\end{tabular}

ção, apresentou boa estimativa da ETo, com erro padrão de 0,44 $\mathrm{mm} \mathrm{d}^{-1}$, obtendo-se um valor médio de ETo próximo ao determinado pelo método de Penman-Monteith, ao contrário, os métodos de Hargreaves-Samani e da Radiação apresentaram erro padrão de estimativa de 1,17 e $0,60 \mathrm{~mm} \mathrm{~d}^{-1}$, respectivamente. Esses métodos superestimaram a evapotranspiração de referência. De acordo com Jensen et al. (1990), os métodos que se baseiam na temperatura do ar e na radiação, caso de Hargreaves-Samani, tendem a superestimar a evapotranspiração de referência em 15 a 25\%, em climas úmidos, resultados similares também foram encontrados por Souza \& Yoder (1994), no interior do Ceará. Quanto ao método da Radiação, seu resultado não foi satisfatório, apresentando alto erro padrão da estimativa, contrariando os resultados obtidos por Oliveira \& Carvalho (1998), nos municípios de Seropédica e Campos, Estado do Rio de Janeiro. Lunardi et al. (1999) verificaram superestimativa da ETo com o uso do método da Radiação em Botucatu, estado de São Paulo, indicando que este método necessita de calibrações locais para estimativa confiável da ETo.

O tanque Classe A apresentou resultados satisfatórios para períodos diários, com erro padrão de $0,62 \mathrm{~mm} \mathrm{~d}^{-1}$, apesar de ser recomendado, de maneira generalizada, para períodos de tempo superiores a dez dias para estimativa da $\mathrm{ET}_{0}$.

O Irrigâmetro indicou valores de evapotranspiração de referência próximos dos obtidos pelo método de Penman-Monteith - FAO 56, com estimativa do erro padrão igual a $0,53 \mathrm{~mm} \mathrm{~d}^{-1}$. A análise conjunta dos valores dos coeficientes angular, de determinação e de ajustamento e da estimativa do erro padrão, indica que o Irrigâmetro foi, dentre os métodos estudados, o que melhor estimou a ETo com base no método padrão, superestimando-a em apenas 5,6\%, sendo assim, o Irrigâmetro mostrou resultados confiáveis de estimativa da ETo para períodos diários que, associados ao baixo custo, à alta praticidade e à dispensa de cálculos adicionais, o tornam indicado para fins de manejo da água de irrigação.

Encontram-se, na Figura 3, as correlações entre valores diários de evapotranspiração de referência estimados pelos métodos estudados e pelo método de Penman-Monteith - FAO 56.

Os métodos de Penman modificado e da Radiação subestimaram a ETo para valores menores que $3 \mathrm{~mm} \mathrm{~d}^{-1}$ e superestimaram para valores maiores. O método de Hargreaves-Samani superestimou a ETo ocorrendo, portanto, comportamento contrário com uso do tanque Classe A. 

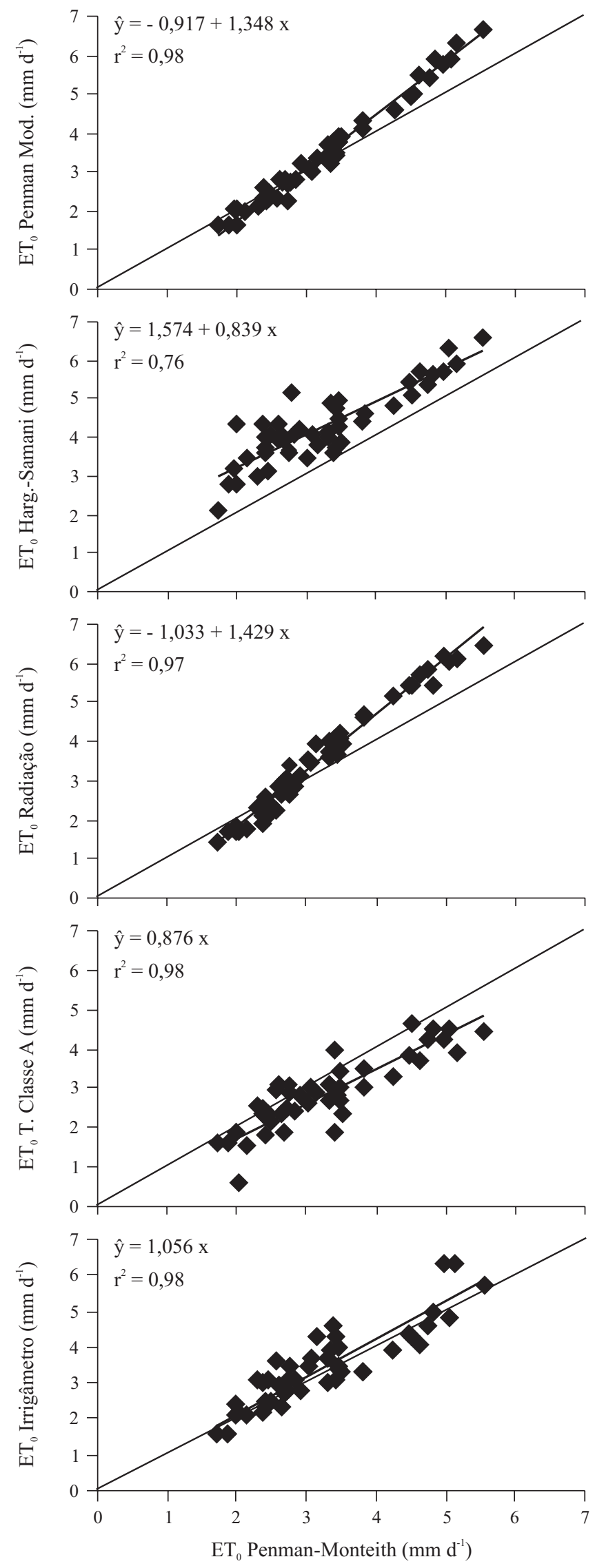

Figura 3. Relação entre os valores de $\mathrm{ET}_{0}$ determinada pelos métodos de Penman modificado, Hargreaves-Samani, Radiação, tanque Classe A e pelo Irrigâmetro, com valores de ETo obtida pelo método de Penman-Monteith - FAO 56, para intervalos de um dia
Observa-se, na Figura 3, que a linha de tendência do Irrigâmetro praticamente acompanha a linha de valores 1:1 apresentando melhor desempenho que os outros métodos considerados neste estudo, tendo por base o método de Penman-Monteith - FAO 56.

As comparações dos métodos nas escalas de três, cinco e sete dias, seguiram a mesma metodologia estatística aplicada na escala diária e estão apresentadas na Tabela 2.

Tabela 2. Parâmetros da regressão, coeficiente de determinação $\left(\mathrm{r}^{2}\right)$, estimativa do erro padrão (EEP) e coeficiente de ajustamento (d), obtidos das correlações entre os valores de ETo $\left(\mathrm{mm} \mathrm{d}^{-1}\right)$ estimada no Irrigâmetro e pelos demais métodos estudados, com valores estimados pelo método de Penman-Monteith - FAO 56, para todos os períodos de tempo estudado

\begin{tabular}{|c|c|c|c|c|c|c|}
\hline Método & $b_{0}=0$ & $b_{1}=1$ & $r^{2}$ & EEP & d & ETo \\
\hline \multicolumn{7}{|c|}{ Período de três dias } \\
\hline P. - Monteith - FA0 56 & & & & & & 3,23 \\
\hline Penman modificado & $-0,933^{*}$ & 1,350 * & 0,99 & 0,35 & 0,96 & 3,43 \\
\hline Hargreaves-Samani & 1,298 * & $0,935^{\mathrm{ns}}$ & 0,85 & 1,16 & 0,67 & 4,32 \\
\hline Radiação & $0,958 *$ & $1,403^{*}$ & 0,97 & 0,51 & 0,93 & 3,57 \\
\hline Tanque Classe A & - & $0,889^{*}$ & 0,99 & 0,46 & 0,89 & 2,89 \\
\hline Irrigâmetro & - & $1,079 *$ & 0,99 & 0,40 & 0,93 & 3,50 \\
\hline \multicolumn{7}{|c|}{ Período de cinco dias } \\
\hline P. - Monteith - FA0 56 & & & & & & 3,26 \\
\hline Penman modificado & $-1,132^{*}$ & $1,442^{*}$ & 0,91 & 0,56 & 0,90 & 3,57 \\
\hline Hargreaves-Samani & $1,004^{*}$ & $1,031^{\mathrm{ns}}$ & 0,99 & 1,20 & 0,67 & 4,36 \\
\hline Radiação & $-0,711^{*}$ & $1,320 *$ & 0,99 & 0,45 & 0,93 & 3,59 \\
\hline Tanque Classe A & - & $0,894^{*}$ & 0,99 & 0,45 & 0,88 & 2,93 \\
\hline Irrigâmetro & - & 1,069 * & 0,99 & 0,35 & 0,94 & 3,49 \\
\hline \multicolumn{7}{|c|}{ Período de sete dias } \\
\hline P. - Monteith - FA0 56 & & & & & & 3,25 \\
\hline Penman modificado & $-0,847^{*}$ & $1,323^{*}$ & 0,99 & 0,32 & 0,96 & 3,46 \\
\hline Hargreaves-Samani & $1,055^{*}$ & $1,012^{\mathrm{ns}}$ & 0,89 & 1,21 & 0,67 & 4,34 \\
\hline Radiação & - & $1,117^{*}$ & 0,99 & 0,48 & 0,93 & 3,60 \\
\hline Tanque Classe A & - & $0,889^{*}$ & 0,99 & 0,42 & 0,90 & 2,90 \\
\hline Irrigâmetro & - & $1,068^{*}$ & 0,99 & 0,28 & 0,97 & 3,48 \\
\hline
\end{tabular}

Tem-se, na Tabela 2, que o agrupamento da ETo em intervalos de três, cinco e sete dias, respectivamente, resultou em aumento dos coeficientes de determinação e de ajuste, com valores mais próximos da unidade, e em redução da estimativa do erro padrão, com valores mais próximos de zero para a maioria dos métodos estudados, comparativamente ao intervalo diário.

Para as escalas de três, cinco e sete dias, o Irrigâmetro apresenta valores da estimativa do erro padrão iguais a 0,40 , 0,35 e $0,28 \mathrm{~mm} \mathrm{~d}^{-1}$ e coeficiente de ajustamento igual a 0,93 , 0,94 e 0,97, respectivamente, indicando melhor desempenho para estimativa da ETo frente aos demais métodos estudados.

As Figuras 4, 5 e 6 apresentam as correlações entre valores de ETo estimados pelos métodos estudados, pelo Irrigâmetro e pelo método de Penman-Monteith - FAO 56, para intervalos de três, cinco e sete dias, respectivamente.

A análise das Figuras 4, 5 e 6 mostra que o agrupamento dos valores de ETo em períodos maiores tende a melhorar a estimativa, haja vista ser mantido o mesmo comportamento dos métodos estudados. O método de Hargreaves-Samani foi, dentre os estudados, o que mais superestimou a ETo, independentemente 

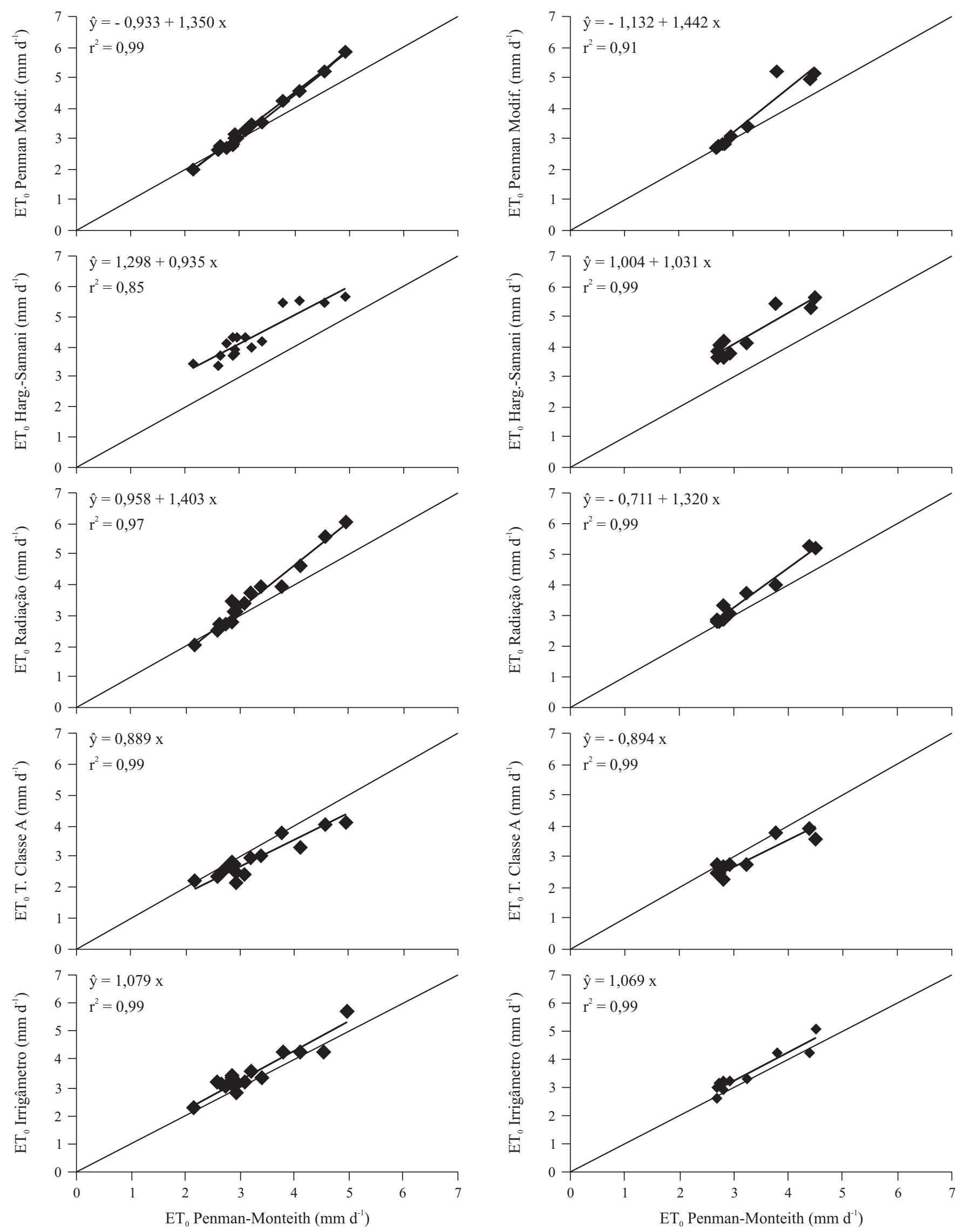

Figura 4. Relação entre os valores de ETo estimados pelos métodos de Penman modificado, Hargreaves-Samani, Radiação, tanque Classe A e pelo Irrigâmetro, com valores de ETo obtidos pelo método de Penman-Monteith - FAO 56, para intervalos de três dias

Figura 5. Relação entre os valores de ETo estimados pelos métodos de Penman modificado, Hargreaves-Samani, Radiação, tanque Classe A e pelo Irrigâmetro, com valores de ETo obtidos pelo método de Penman-Monteith - FAO 56, para intervalos de cinco dias 

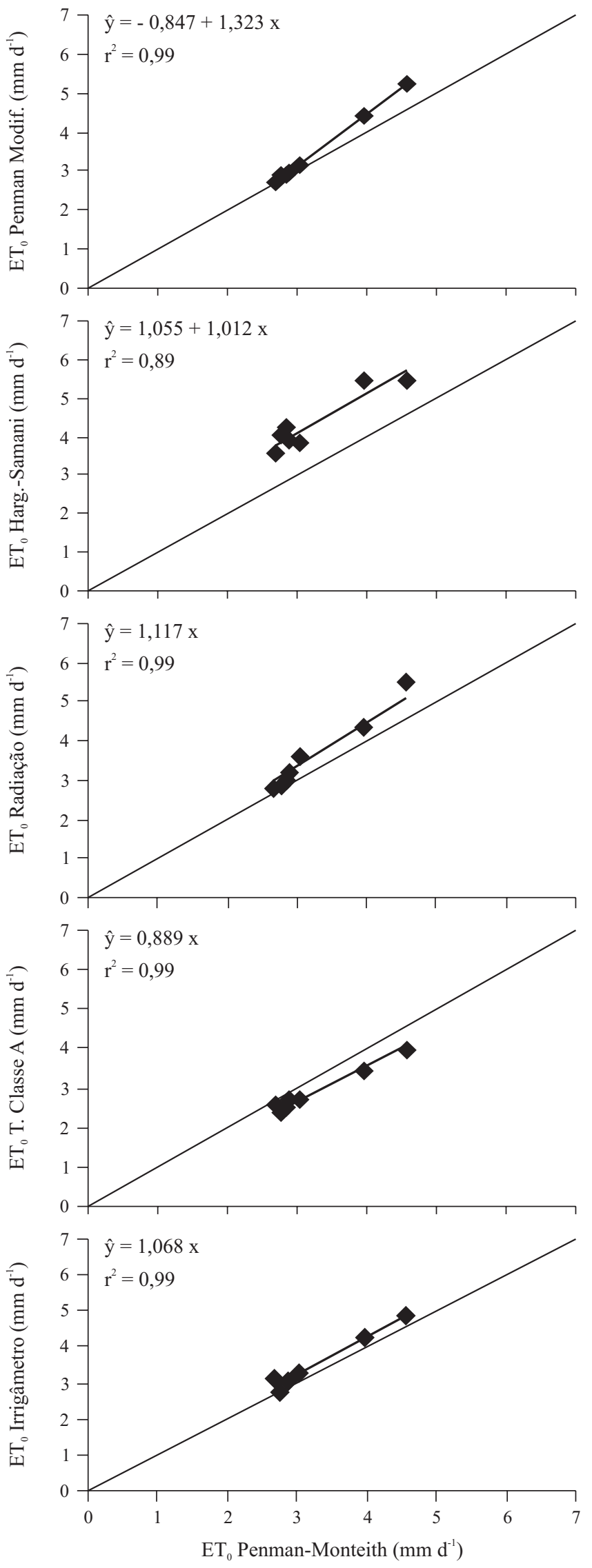

Figura 6. Relação entre os valores de ETo estimados pelos métodos de Penman modificado, Hargreaves-Samani, Radiação, tanque Classe A e pelo Irrigâmetro, com valores de ETo obtidos pelo método de Penman-Monteith - FAO 56, para intervalos de sete dias do intervalo de comparação. Jensen et al. (1990) encontraram boas estimativas de ETo pelo método de Hargreaves-Samani para intervalos de tempo maiores que dez dias.

O tanque Classe A apresentou melhoria na estimativa da ETo para intervalos de sete dias com maior precisão em relação aos demais períodos analisados (Jensen et al.,1990; Doorenbos \& Pruitt, 1977).

Nos intervalos de tempo analisados, o Irrigâmetro mostrou valores médios de evapotranspiração de referência praticamente iguais aos valores obtidos pelo método padrão, tornando-o indicado para o manejo da água na agricultura irrigada.

\section{CONCLUSÕES}

1. O irrigâmetro apresentou desempenho satisfatório para estimar a ETo nas escalas de um, três, cinco e sete dias, fato que, associado ao baixo custo, à alta praticidade e à dispensa de cálculos adicionais, o indica para o manejo da água na agricultura irrigada.

2. O método de Penman modificado subestimou a ETo obtida pelo método de Penman-Monteith - FAO 56, para valores menores que $3 \mathrm{~mm} \mathrm{~d}^{-1}$, apresentando comportamento contrário para valores maiores.

3. Os métodos de Hargreaves-Samani e da Radiação superestimaram a ETo obtida pelo método de Penman-Monteith - FAO 56, em todos os intervalos de tempo analisados, tendo o tanque Classe A comportamento contrário.

\section{LITERATURA CITADA}

Allen, R. G. A Penman for all seasons. Journal of Irrigation and Drainage Engineering, v.112, n.4, p.348-386, 1986.

Allen, R. G. REF-ET: Reference evapotranspiration calculator, Version 2.1.Idaho: Idaho University, 2000. 82p.

Allen, R. G.; Pereira, L. S.; Raes, D.; Smith, M. Guidelines for computing crop water requirements. Rome: FAO, 1998. 310p. Irrigation and Drainage Paper, 56.

Doorenbos, J.; Pruitt, J. O. Crop water requirement. Rome: FAO, 1977. 144p. Irrigation and Drainage Paper 24.

Hargreaves, G. H.; Samani, Z. A. Reference crop evapotranspiration from temperature. Applied Engineering Agriculture, v.1, n.2, p.96-99, 1985.

Jensen, M. E.; Burman, R. D.; Allen, R. G. Evapotranspiration and irrigation water requirements. New York: ASCE, 1990. 332p.

Ley, T. W.; Hill, R. W.; Jensen, D. T. Errors in Penman-Wright alfalfa reference evapotranspiration estimates: I. Model sensitivity analyses. Transaction of ASAE, v.37, n.6, p.1853-1861, 1994a.

Ley, T. W.; Hill, R. W.; Jensen, D. T. Errors in Penman-Wright alfalfa reference evapotranspiration estimates: II. Effects of weather sensor measurement variability. Transaction of ASAE, v.37, n.6, p.1863-1870, 1994b.

Lunardi, M. A.; Lunardi, D. M. C.; Cavati, N. Comparação entre medidas evapotranspirométricas e metodológicas da FAO, na determinação da evapotranspiração de referência. IRRIGA, v.4, n.1, p.52-66, 1999. 
Oliveira, M. A. A. de; Carvalho, D. F. de. Estimativa da evapotranspiração de referência e da demanda suplementar de irrigação para o milho (Zea mays L.) em Seropédica e Campos, estado do Rio de Janeiro. Revista Brasileira de Engenharia Agrícola e Ambiental, v.2, n.2, p.132-135, 1998.

Oliveira, R. A. de; Tagliaferre, C. Irrigâmetro: nova tecnologia para manejo da água de irrigação. In: Barbosa, T. C.; Taniguchi, G. C.; Penteado, D. C. S.; Silva, D. J. H. da. Ambiente protegido: Olericultura, citricultura e floricultura. Viçosa: UFV, 2006, p.39-64.
Sediyama, G. C. Necessidade de água para os cultivos. Brasília: ABEAS, 1996. 176p.

Smith, M. Report on the expert consultation on revision of crop water requirements. Rome: FAO, 1991. 45p.

Souza, F. de; Yoder, R. ET estimation in the Northeast of Brazil: Hargreaves or Penman-Monteith equation. ST. Joseph: ASAE, 1994. 6p. Paper, 942545.

Willmott, C. J.; Ckleson, S. G.; Davis, R. E. Statistics for evaluation and comparisons of models. Journal of Geophysical Research, v.90, n.C5. p.8995-9005, 1985. 